\title{
AREA EFFICIENT 3.3GHz PHASE LOCKED LOOP WITH FOUR MULTIPLE OUTPUT USING 45NM VLSI TECHNOLOGY
}

\author{
Ms. Ujwala A. Belorkar ${ }^{1}$ and Dr. S.A.Ladhake ${ }^{2}$ \\ ${ }^{1}$ Department of electronics \& telecommunication ,Hanuman Vyayam Prasarak Mandal's \\ College of Engineering \& Technology, Amravati. Maharashtra. \\ ujwalabelorkararediffmail. com \\ ${ }^{2}$ Sipana's College of Engineering \& Technology, Amravati, Maharashtra. \\ sladhake@yahoo.co.in
}

\begin{abstract}
This paper present area efficient layout designs for 3.3GigaHertz (GHz) Phase Locked loop (PLL) with four multiple output. Effort has been taken to design Low Power Phase locked loop with multiple output, using VLSI technology. VLSI Technology includes process design, trends, chip fabrication, real circuit parameters, circuit design, electrical characteristics, configuration building blocks, switching circuitry, translation onto silicon, CAD and practical experience in layout design. The proposed PLL is designed using $45 \mathrm{~nm}$ CMOS/VLSI technology with microwind 3.1. This software allows designing and simulating an integrated circuit at physical description level. The main novelties related to the $45 \mathrm{~nm}$ technology are the high-k gate oxide, metal gate and very low-k interconnect dielectric. The effective gate length required for $45 \mathrm{~nm}$ technology is $25 \mathrm{~nm}$. Low Power (0.211miliwatt) phase locked loop with four multiple outputs as PLL8x, PLL4x, PLL2x, \& PLL1x of $3.3 \mathrm{GHz}, 1.65 \mathrm{GHz}, 0.825 \mathrm{GHz}$, and $0.412 \mathrm{GHz}$ respectively is obtained using $45 \mathrm{~nm}$ VLSI technology.
\end{abstract}

Index Terms : phase-locked loop (PLL), high performance voltage-controlled oscillator (VCO), $45 \mathrm{~nm}$ technology, multiple outputs, low power.

\section{INTRODUCTION}

Power has become one of the most important paradigms of design convergence for multi gigahertz communication systems such as optical data links, wireless products, microprocessor \& ASIC/SOC designs. The current leading-edge technologies (such as low bit-rate video and cellular communications) already provide the end-users a certain amount of processing power and portability. This trend is expected to continue, with very important implications on VLSI and systems design. One of the most important characteristics of information services is their increasing need for very high processing power and bandwidth (in order to handle real-time video, for example). The other important characteristic is that the information services tend to become more and more personalized (as opposed to collective services such as broadcasting), which means that the devices must be more intelligent to answer individual demands, and at the same time they must be portable to allow more flexibility/mobility.

Since the multiple outputs Phase Locked Loop (PLL) provides multiple clock generation, it is to be needed to design PLL with multiple output for modern communication Engineering applications with low power, high stability and low jitter. 
This paper introduces a design aspect for layout design of low power PLL with four multiple output using VLSI technology.

PLL is widely applied for different purposes in various domains such as communication and instrumentation. Phase locked loop can be used to maintain a well-defined phase, and hence frequency relation between two independent signal sources [2].

The proposed PLL is a feed back system composed of three elements: a phase detector, a loop filter and a high performance voltage controlled oscillator (VCO).

Reported work describes the use of VLSI technology to design optimum layout for Low Power, High performance phase locked loop with multiple output. The design process, at various levels, is usually evolutionary in nature. It starts with a given set of requirement. When the requirements are not met, the design has to be improved. More simplified view of the VLSI technology consists of various representations, abstractions of design, logic circuits, CMOS circuits and physical layout.

Here for the design, microwind 3.1 VLSI Backend software is used. This software allows designing and simulating an integrated circuit at physical description level. The proposed PLL is designed using $45 \mathrm{~mm}$ CMOS/VLSI technology in microwind 3.1software, which in turn offers high speed performance at low power [1].

The main novelties related to the $45 \mathrm{~nm}$ technology are the high-k gate oxide, metal gate and very low-k interconnect dielectric [1]. The effective gate length required for $45 \mathrm{~nm}$ technology is $25 \mathrm{~nm}$. Some of the key features of $45 \mathrm{~nm}$ technologies from various providers like TSMC, Fujitsu, and Intel are as given in table-I below.

\section{TABLE I}

\section{Key Features OF 45 NM TeChNOLOGY}

\begin{tabular}{|c|c|}
\hline Parameter & Value \\
\hline VDD (V) & $0.85-1.2 \mathrm{~V}$. \\
\hline Ioff N (nA/um) & $5-100$ \\
\hline Ioff P (nA/um) & $5-100$ \\
\hline Gate dielectric & ${\mathrm{SiON}, \mathrm{HfO}_{2}}$ \\
\hline No. of metal layers & $6-10$ \\
\hline
\end{tabular}

Compared to $65-\mathrm{nm}$ technology, $45 \mathrm{~nm}$ technology must offer:

1) $30 \%$ increases in switching performance

2) $30 \%$ reduction in Power consumption

3) 2 times higher density 
4) 2 times reduction of the leakage between source and drain and through the gate oxide [1].

Considering the advantage of $45 \mathrm{~nm}$ technologies over $90 \mathrm{~nm} \& 65 \mathrm{~nm}$ technologies, the proposed work is done with $45 \mathrm{~nm}$ technologies. Power consumption is a limiting factor in VLSI integration for portable applications. The resulting heat dissipation also limits the feasible packaging and performance of the VLSI chip. Since the dynamic power dissipation in synchronous digital integrated circuit is determined by $C V^{2} f$, reducing the supply voltage is an effective way to reduce power consumption of the modern electronic systems [2]. As the supply voltage scales down with the technology, any power supply noise on power and ground level affects the analog circuit performance more than before. This power supply noise has a direct effect on the voltage controller oscillator (VCO) output frequency of PLL which is proportional to the control voltage from the charge pump.

Following steps are involved to obtain the proposed design. Every step of design follows the design flow of microwind 3.1 software.

\section{Proposed Phase Locked loop Design}

Until DSP technology is capable of directly processing and generating the RF signals used to transmit wireless data, raditional RF engineering will remain a fundamental part of wireless communication systems design. As it stands, wireless transceivers must still be able to generate a wide range of frequencies in order to upconvert the outgoing data for transmission and downconvert the received signal for processing. Monolithic phase locked loops have been used for clock- $\&$-data recovery in communication system, clock generation and distribution in microprocessor and frequency synthesis in wireless application. [3].

A proposed PLL is a feedback system composed of three elements: a phase detector, a loop filter and a high performance voltage controlled oscillator (VCO). To obtain the layout of proposed PLL, CMOS circuit of each element of proposed PLL is converted into physical layout using lamda based rules of microwind 3.1 software. After cascading the layout of each element, final layout is obtained. This paper particularly focuses on analysis and design of phase-locked loop with low power consumption using VLSI technology.

\section{DESIGN OF PHASE DETECTOR \& FILTER}

The phase detector of the PLL is the XOR gate [3]. The XOR gate output produces a regular square oscillation $\mathrm{V}_{\mathrm{PD}}$ when the clock input circuit and signal input clkIn have one quarter of period shift $\left(90^{\circ}\right.$ or $\left.\pi / 2\right)$. 
Average value Of VPD

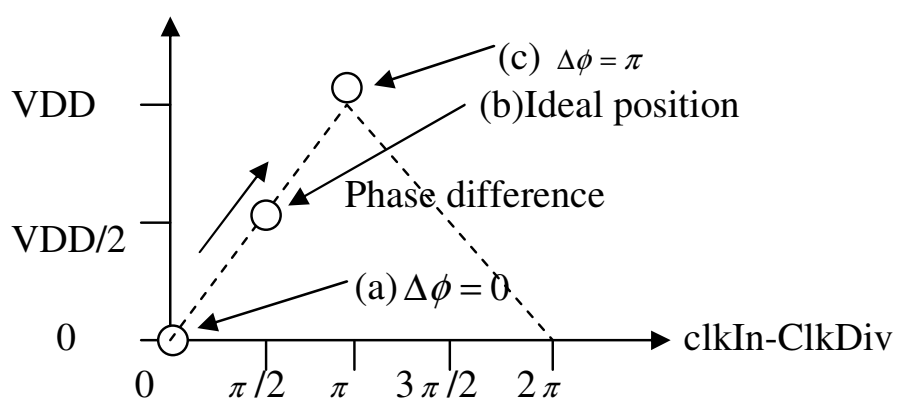

Fig.2.2.1 The XOR Phase detector at work

For other angles, the output is no more regular. As shown in fig-1, at initialization, the average value of XOR output $\mathrm{V}_{\mathrm{PD}}$ is close to 0 . When the phase between clkDiv and ClkIn is around $\pi / 2, V_{P D}$ is $\mathrm{VDD} / 2$, then it increases to VDD.

The gain of the phase detector is the ratio between $V_{P D}$ and $\Delta \phi$. When the phase between clkDiv and ClkIn is around $\pi / 2, V_{P D}$ is VDD/2, then it increases to VDD. The gain of the phase detector is the ratio between $V_{P D}$ and $\Delta \phi$. When the phase difference is larger than $\pi$, the slope sign is negative until $2 \pi$. When locked, the phase difference should be close to $\pi / 2$.

The XOR gate output produces a regulator square oscillation VPD when the clkIn and the signal clkdiv have one quarter of period shift ( 90 or $\Pi / 2$ ). For other angles output is not regular. Fallowing fig - 2 shows CMOS circuit for XOR gate.

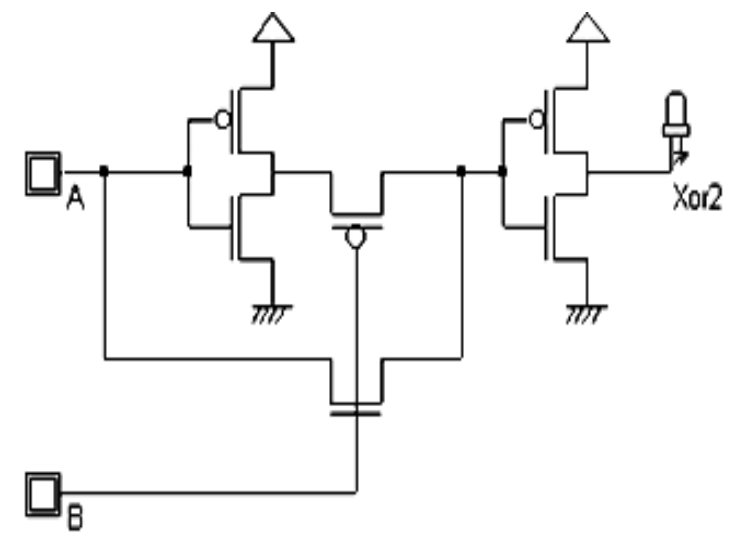

Fig. 2 CMOS circuit for XOR Gate 
The low-pass filter smooth out the abrupt control inputs from the charge pump. Since initially the oscillator may be far from the reference frequency, practical phase detectors may also respond to frequency differences, so as to increase the lock-in range of allowable inputs. The low pass filter for proposed PLL is designed using virtual resistance of $1000 \mathrm{ohm}$ with capacitor of 2 Pico farad.

\section{Design of the Voltage Controlled Oscillator And Multiple OUTPUT UNIT}

The VCO is the most important functional unit in the PLL. Its output frequency determines the effectiveness of PLL. In addition to operating at highest frequency, this unit consumes the most of the power in the system.

Obviously, this unit is of particular focus to reduce power consumption. PLL with multiple outputs means to VCO with multiple output. Voltage Controlled Oscillator required for PLL should posses following characteristics.

1) The oscillating frequency should be restricted to the required bandwidth. For example, in European mobile phone applications, the VCO frequency should be varying between flow $=1700 \mathrm{MHz}$ and $\mathrm{High}=1800 \mathrm{MHz}$ [3].

2) Due to process variations, the VCO frequency range should be extended to fmin, fmax, typically $10 \%$ higher and lower than the request range.

3) When the control voltage $\mathrm{Vc}_{\mathrm{c}}$ is equal to $\mathrm{V}_{\mathrm{DD}} / 2$, the clock should be centered in the middle of the desired frequency range.

4) The duty cycle of VCO clock output should be as close as possible to $50 \%$. If this is not the case, the PLL would have problems locking, or would not produce a stable output clock [3].

\section{A. High Performance VCO}

The proposed PLL uses high performance VCO as shown in fig-3. It provides very good linearity. The principle of this VCO is a delay cell with linear delay dependence on the control voltage. The delay cell consists of a p-channel MOS (pmos) in series, controlled by Vcontrol, and a pull-down n-channel MOS (nmos) controlled by Vplage. The delay dependence on Vcontrol is almost linear for the fall edge. The key point is to design an inverter just after the delay-cell with a very low commutation point Vc. The rise edge is almost unchanged [3].

The delay cells are connected, to delay both rise and fall edge of the oscillator as shown in following figure-3. 


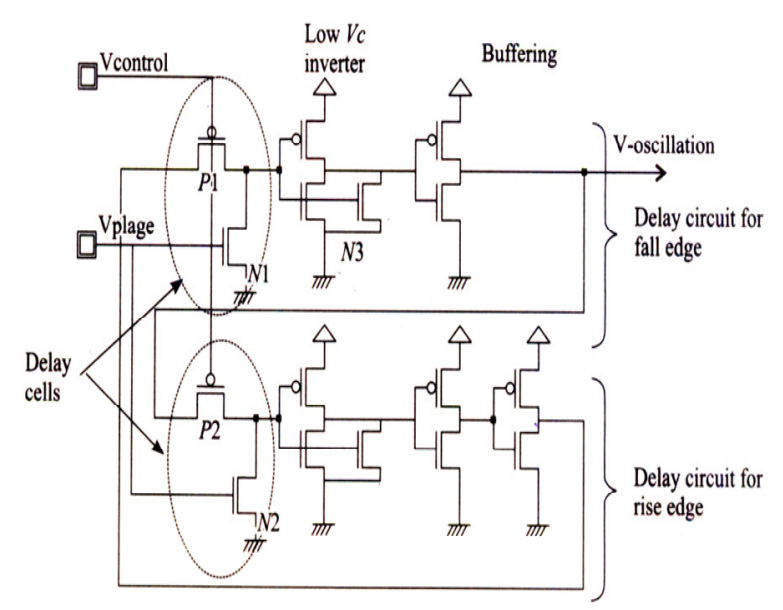

Fig. 3 High performance VCO

\section{B. Multiple Outputs PLL}

For multiple output PLL, 1x is the basic output of PLL, the $90^{\circ}$ phase shifted output is generated using delay circuits or using logic circuits which in turn offers his speed performance at low power. It have multiple outputs $1 \mathrm{x}, 2 \mathrm{x}, 4 \mathrm{x}$ and $8 \mathrm{x}$, which can be utilized in multiphase clocking circuits.

Figure-5 shows the block schematic of multiple output (four output) PLL with high performance VCO.

To achieve the proposed target fallowing steps are include in the design and analysis of proposed PLL.

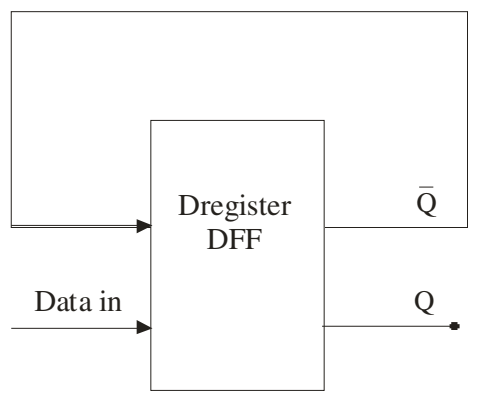

\section{Fig. 4 Block of Dregister ( DFF )}

1) Schematic design of each block of proposed PLL using CMOS transistors.

2) Performance verification of the above for different parameters.

3) CMOS layout for the proposed PLL using Lamda defined rules of VLSI backend3.1 software. 
4) Verification of CMOS layout and parameter testing.

5) If the goal is achieved for all proposed parameter including detail verification, sing off for the design analysis and design will be ready for IC making.

6) If detail verification of parameters would not completed then again fallow the first step with different methodology.

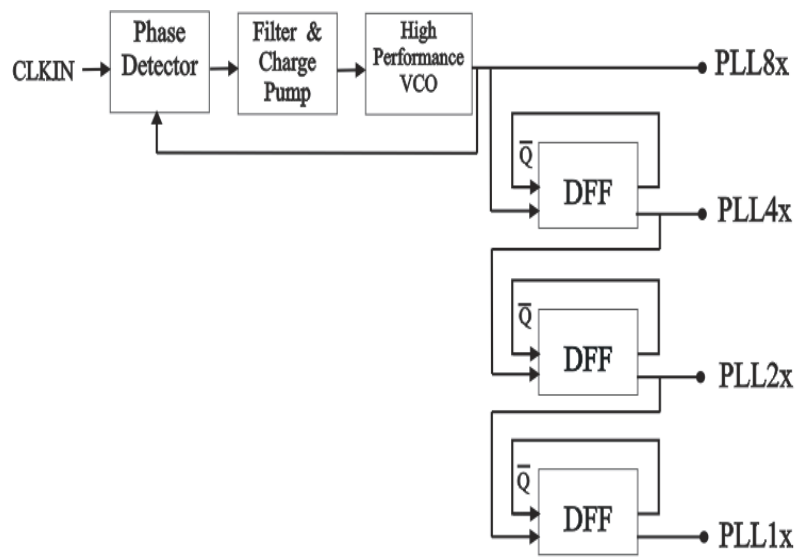

Fig. 5 Block schematic of PLL with four outputs

As a result of technology scaling, there are increased process variations of circuit parameters such as the transistor channel length and transistor threshold voltage. The increased process variations can have a significant effect on circuit performance and power variations also have an impact on how exactly a parallel system should be designed [4]. BSIM4 model of the transistor nmos and pmos is having the specifications as fallows:

N-MOS BSIM4:

low leakage

MODEL N1 NMOS LEVEL $=14$ VTHO $=0.19$

$\mathrm{U} 0=0.020 \mathrm{TOXE}=3.5 \mathrm{E}-9 \mathrm{LINT}=0.000 \mathrm{U}$ 


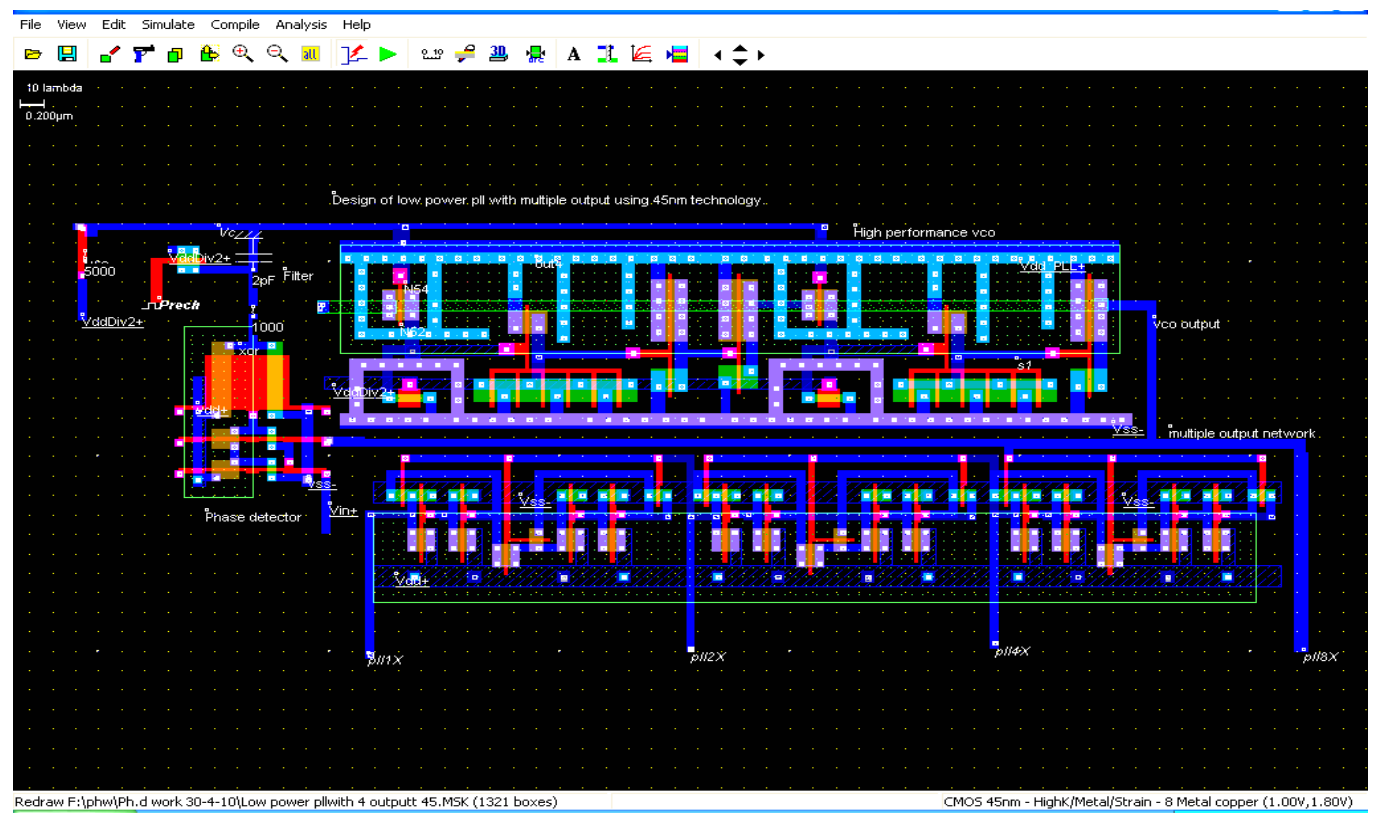

Fig. 6 Layout Design of PLL with four output

$\mathrm{K} 1=0.750 \mathrm{~K} 2=0.100 \mathrm{DVT} 0=2.300$

DVT1 $=0.540$ LPE0 $=2.200 \mathrm{e}-9$ ETA0 $=0.080$

NFACTOR $=0.1 \mathrm{U} 0=0.020 \mathrm{UA}=6.300 \mathrm{e}-15$

WINT $=0.020 \mathrm{U}$ LPE0 $=2.200 \mathrm{e}-9$

$\mathrm{KT} 1=-0.060 \mathrm{UTE}=-1.800 \mathrm{VOFF}=0.000$

$\mathrm{XJ}=0.150 \mathrm{U}$ NDEP $=170.000 \mathrm{e} 15 \mathrm{PCLM}=1.100$

$\mathrm{CGSO}=100.0 \mathrm{p} \mathrm{CGDO}=100.0 \mathrm{p}$

$\mathrm{CGBO}=60.0 \mathrm{p}$

P-MOS BSIM4:

Low leakage

MODEL P1 PMOS LEVEL $=14$ VTHO $=-0.15$ U0 $=0.018$ TOXE $=3.5 E-9$ LINT $=0.000 \mathrm{U}$

$\mathrm{K} 1=0.650 \mathrm{~K} 2=0.100 \mathrm{DVT} 0=2.300$

DVT $1=0.540$ LPE0 $=2.200 \mathrm{e}-9$ ETA0 $=0.080$

NFACTOR $=0.1 \mathrm{U} 0=0.018 \mathrm{UA}=9.500 \mathrm{e}-15$

WINT $=0.020 \mathrm{U}$ LPE0 $=2.200 \mathrm{e}-9$

$\mathrm{KT} 1=-0.060 \mathrm{UTE}=-1.800 \mathrm{VOFF}=0.000$

$\mathrm{XJ}=0.150 \mathrm{U}$ NDEP $=170.000 \mathrm{e} 15 \mathrm{PCLM}=0.700$

$\mathrm{CGSO}=100.0 \mathrm{p} \mathrm{CGDO}=100.0 \mathrm{p}$

$\mathrm{CGBO}=60.0 \mathrm{p}$ 


\section{Simulation Results}

Figure.6 shows the optimum, high efficient chip design of low power PLL with four multiple output using 45nm VLSI technology. This layout design is implemented using 29 nmos along with 28 pmos BSIM4 transistors with optimum dimensions of transistors and metal connections fallowing the lamda based rules of microwind 3.1 software. This phase locked loop give a four multiple output as PLL8x equal to $3.3 \mathrm{GHz}$, PLL4x equal to $1.65, \mathrm{GHz}$ PLL $2 x$ equal to $0.825 \mathrm{GHz}$ and PLL $1 \mathrm{x}$ equal to $0.412 \mathrm{GHz}$..

\section{TABLE II}

\section{Analysis Of Voltage Variation Of VdD On Frequency Of VCo OUTPUT PLL1X}

\begin{tabular}{|c|c|}
\hline VDD Volt & Freq. (GHz) \\
\hline $0.800 \mathrm{~V}$ & 0.416 \\
\hline $0.900 \mathrm{~V}$ & 0.416 \\
\hline $1.00 \mathrm{~V}$ & 0.416 \\
\hline $1.10 \mathrm{~V}$ & 0.416 \\
\hline $1.20 \mathrm{~V}$ & 0.416 \\
\hline
\end{tabular}

TABLE III

\section{Analysis Of Voltage VARIATion Of VdD On Frequency Of VCo OUTPUT} PLL8X

\begin{tabular}{|c|c|}
\hline VDD Volt & Freq. (GHz) \\
\hline $0.800 \mathrm{~V}$ & 3.306 \\
\hline $0.900 \mathrm{~V}$ & 3.306 \\
\hline $1.00 \mathrm{~V}$ & 3.306 \\
\hline $1.10 \mathrm{~V}$ & 3.306 \\
\hline $1.20 \mathrm{~V}$ & 3.306 \\
\hline
\end{tabular}

Figure -7 shows the frequency versus time response of PLL with four multiple output. It shows that as control voltage Vc increases the frequency of PLL decreases. 


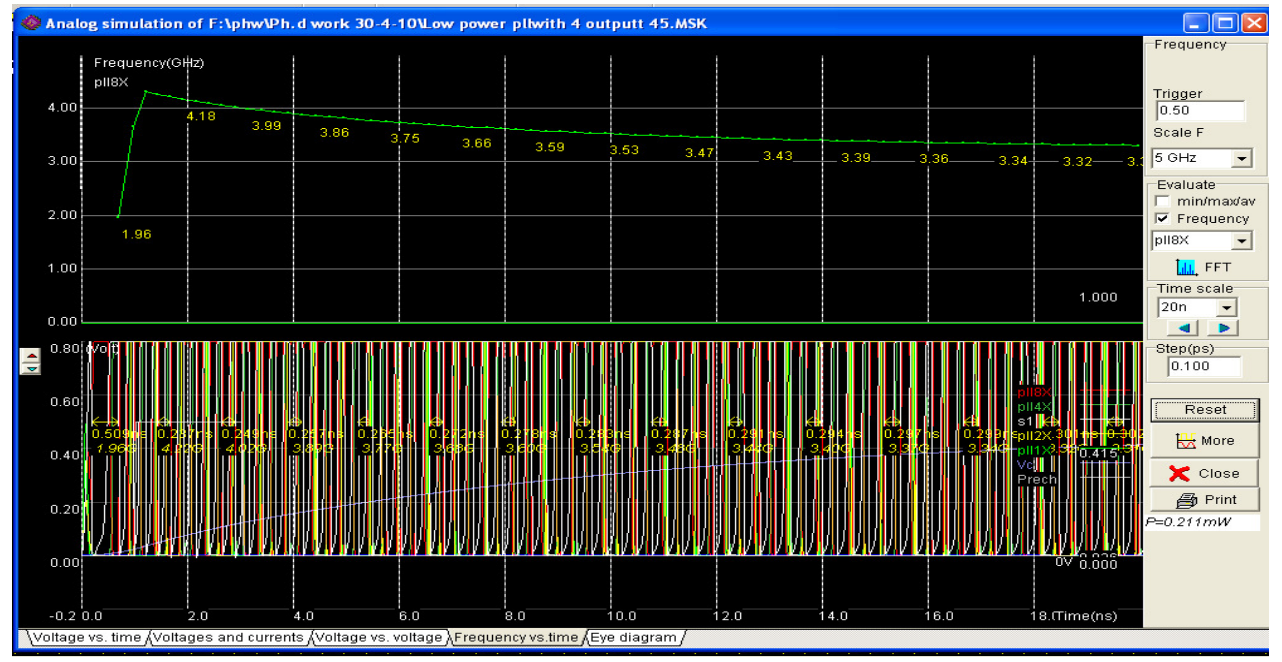

Fig. 7 Frequencies vs. time response of proposed PLL

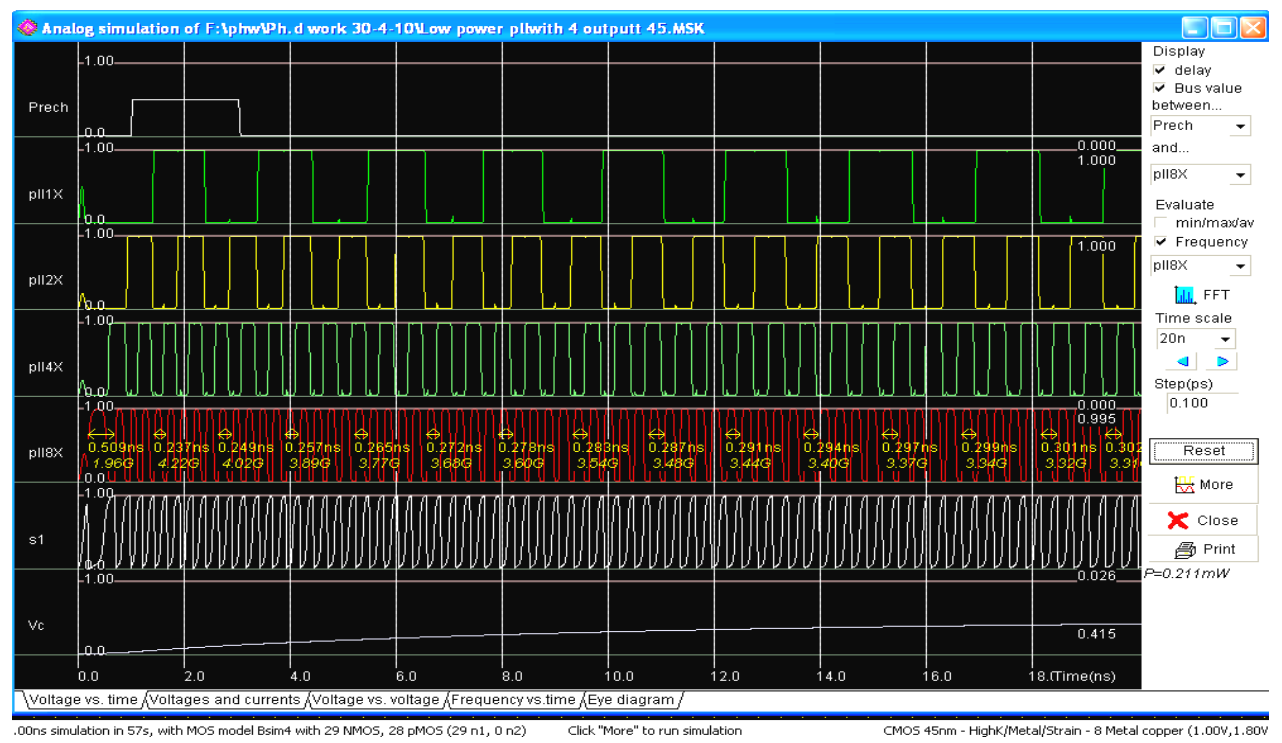

Fig. 8 Voltage vs. time wave forms of PLL with four multiple output

The PLL is locked at $3.3 \mathrm{GHz}$ frequency. Figure- 8 shows voltage verses time waveforms of PLL's outputs and inputs. When control voltage is reaches to 0.415 volt, PLL is locked to the frequency of $3.3 \mathrm{GHz}$. Multiple output of the PLL gives multiple frequencies of amplitude 1volt.

Above table II \& III shows that, though there is variation of supply $\mathrm{V}_{\mathrm{DD}}$ from $0.8 \mathrm{~V}$ to $1.2 \mathrm{~V}$, the output of PLL remained stable which proved the high stability of the PLL. From the parametric analysis of designed PLL the power dissipation measured by $\mathrm{V}_{\mathrm{DD}}$ at $1 \mathrm{~V}$ is found to be $0.211 \mathrm{mw}$, which shows that power consumption is very low (Low power). 
Thus the effort has been taken to design high efficient, optimum area chip for PLL with four outputs.

\section{CONCLUSION}

The proposed PLL is designed using $45 \mathrm{~nm}$ CMOS/VLSI technology with microwind 3.1. This PLL gives a four multiple outputs as PLL8x, PLL4x, PLL2x and PLL1 $x$ simultaneously. This can be used for multichanneling communication system which in turn provides a very fast multitasking communication. This layout design is implemented using 29 nmos along with 28 pmos BSIM4 transistors. Though there is the variation of supply voltage $\mathrm{V}_{\mathrm{DD}}$ from $0.8 \mathrm{~V}$ to $1.2 \mathrm{~V}$, all the output of proposed PLL is found stable which proves the high stability of the PLL. From the parametric analysis of design tool, the power dissipation measured by $\mathrm{V}_{\mathrm{DD}}$ at 1 Volt is found 0.211 miliwatt, which shows that power consumption is very low. In this way very high efficient, low power optimum area chip is designed for phase locked loop with four multiple outputs as PLL8x, PLL4x, and PLL2x \& PLL1x of $3.3 \mathrm{GHz} 1.65 \mathrm{GHz}, 0.825 \mathrm{GHz}$, and 0.412 $\mathrm{GHz}$ respectively.

\section{ACKNOWLEDGEMENT}

The authors wish to thank Vinay Sharma, Director, Ni2 logic design Pvt. Ltd., for simulating discussions

\section{REFERENCES}

[1] E. Sicard, Syed Mahfuzul Aziz, “introducing $45 \mathrm{~nm}$ technology in Microwind3,” microwind application note.

[2] Navid Azizi, Student Member, IEEE, Muhammad M. Khellah, Member, IEEE, Vivek K. De, Senior Member, IEEE, and Farid N. Najm, Fellow, IEEE,"Aware Low-Power Design and Block." IEEE transactions on very large scale integration (vlsi) systems, vol. 15, no. 7, july 2007

[3] E. Sicard, S. Delman- Bendhia, "Deep submicron CMOS Design".

[4] S. Borkar, T. Karnik, S. Narendra, T. Tschanz, A. Keshavarzi, and V. De, "Parameter variations and impact on circuits and microarchitecture," in Proc. Design Autom. Conf., 2003, pp. 338-342.

[5] Fernando Rangel De Sousa, "A reconfigurable high frequency phase-locked loop" IEEE transactions on instrumentation \& measurement Vol. 53 No. 4 Aug. 2004.

[6] Recardo Gonzalez, "Supply and threshold voltage scaling for low power CMOS" IEEE journal of solid state circuits Vol. 32 No. 8 April 1997.

[7] Zuoding Wang, "An Analysis of Charge-Pump Phase-Locked Loops" IE EE transactions on circuits and systemsi: regular papers, vol. 52, no. 10, October 2005.

[8] Oscal T. - C. Chen "A power efficient wide range phase locked loop" IEEE journal of solid state circuits vol. 37 No. 1 January 2002.

[9] R. E. Best, "Phase locked loops design, simulation and application", Mc Graw Hill 2003, ISBMO-07-14/20/8.

[10] Y. Eken and J. Uyemura, "A 5.9GHz Voltage Controlled Ring in 0.18- $\mu \mathrm{m}$ CMOS," IEEE Journal of Solid State Circuits, vol. 39, no. 1, Jan. 1997, pp. 230-233. 